\title{
Kala-azar (Visceral Leishmaniasis) Elimination in Bangladesh: Successes and Challenges
}

\author{
Be-Nazir Ahmed • Shah Golam Nabi • Mizanur Rahman • Shahjada Selim • Ariful Bashar • \\ Md. Mahbubur Rashid • Fahima Yeasmin Lira • Tanveer Ahmed Choudhury • \\ Dinesh Mondal
}

Published online: 1 August 2014

(C) Springer International Publishing AG 2014

\begin{abstract}
Visceral leishmaniasis (VL) also known as kalaazar is a major public health problem in Bangladesh. A national VL elimination program was initiated in 2008 in Bangladesh after the signing of a memorandum of understanding between the Government of Bangladesh, India, and Nepal in 2005 for the elimination of VL from these three countries by 2015 . Following the strategic plan of the VL elimination program of the three countries, the national program in Bangladesh was established in 2008. Based on information in the directorate general of health services, expert opinions in a recently held advocacy meeting in Dhaka and on available scientific literature, we report here the successes and challenges of the national VL elimination program in Bangladesh. The program prepared the national kala-azar elimination guidelines and strategic plan for VL elimination in consultation with the technical working group for VL elimination and VL experts in Bangladesh and abroad, including the World Health Organization-The Special Programme for Research
\end{abstract}

B.-N. Ahmed · S. G. Nabi • M. M. Rashid • F. Y. Lira •

T. A. Choudhury

Disease Control, Directorate General of Health Services,

Government of Bangladesh, Mohakhali, Dhaka-1212, Bangladesh

A. Bashar

Suriya Kanta Kala-azar Research Center, Mymensingh, Bangladesh

D. Mondal $(\bowtie)$

Centre For Nutrition and Food Security, Parasitology Laboratory, International Centre for Diarrhoeal Disease Research, Mohakhali, Dhaka 1212, Bangladesh

e-mail: din63d@icddrb.org

M. Rahman

Vector Borne Disease, WHO, Bangladesh, Gulshan 2, Dhaka-1212, Bangladesh

S. Selim

Department of Medicine, Shaheed Suhrawardy Medical College, Sher-e-Bangla Nagor, Dhaka 1207, Bangladesh and Training in Tropical Diseases (TDR). The program trained health staff from all VL endemic hospitals in proper diagnosis and treatment, stratified the country according to VL burden, and introduced the rapid diagnostic test and oral treatment with miltefosine at no cost for patients. Integrated vector control management with indoor residual spraying and the distribution of commercial, long-lasting, insecticidetreated bed-nets were also studied and then implemented. VL burden has declined, but is still far from the target of VL elimination. Thus, the program must continue to maintain these activities and also introduce new activities to involve community participation in the program. The program is facing challenges regarding the shortage of human resources and logistics because of a scarcity of resources. To maintain the success of the program, support from national and international donor agencies and policy makers will be necessary. Other options for the treatment of VL patients as well as for vector control must also be considered.

Keywords Visceral leishmaniasis · National program · Bangladesh $\cdot$ VL $\cdot$ Protozoa $\cdot$ Leishmnaniasis

\section{Introduction}

Visceral leishmaniasis (VL) also known as kala-azar is a public health problem in 90 countries of the world [1]. The estimated annual VL incidence in the world is $0.2-0.4$ million with a $10 \%$ fatality rate [1]. Ninety percent of all VL cases occur in India, Bangladesh, Nepal, Sudan, South Sudan, and Brazil [1]. VL in the Indian sub-continent has unique epidemiologic and etiologic features as follows: VL is localized to the bordering districts of Nepal, India, and Bangladesh; VL is only caused by the Leishmania donovani parasite; there is only one vector, Phlebotomus argentipes; there is a high treatment response to anti-leishmania drugs such as 
miltefosine, liposomal amphotericin B, paromomycin, and amphotericin B deoxycholate; and VL can be diagnosed with the rK39 rapid test in the field [2,3]. These features specific to the Indian sub-continent inspired the policy makers from Bangladesh, India, and Nepal to initiate a national VL elimination program, and a Memorandum of Understanding was signed by the governments of these three countries in 2005 to eliminate VL by 2015 [4]. The elimination target is to reduce VL burden to less than 1 per 10,000 people at the district level in Nepal and at sub-district (upazila) level in India and Bangladesh [4]. The strategic objectives of the elimination program are: (1) early diagnosis and complete case management; (2) integrated vector management (IVM); (3) effective disease surveillance through passive and active case detection; (4) social mobilization and building partnerships; and (5) clinical and operational research [4].

Almost 7 years have passed since the initiation of the VL elimination program in the Indian sub-continent and although the $\mathrm{MoU}$ was signed in 2005 , the VL elimination program was started in Bangladesh in 2008. Here, we report the successes and challenges of the program in Bangladesh following the strategic framework for elimination of VL from the SouthEast Asia region.

The method for this review included VL surveillance data of the Disease Control Unit of the Directorate General of Health Services, the Government of Bangladesh, national and international expert opinion on VL control in Bangladesh from a recently held advocacy meeting on VL in November 2012 in Dhaka (organized by the Disease Control Unit of the Directorate General of Health Services, Bangladesh and Program For Appropriate Technology in Health (PATH)), and information from the published literature.

\section{Early Diagnosis and Complete Case Management}

Bangladesh initiated the elimination program in 2008 with the preparation of national VL elimination guidelines and a country-specific strategic plan for VL elimination by the national technical working group for VL elimination. This was approved by the national steering committee for $\mathrm{VL}$ elimination. Public health staff from the VL endemic subdistricts hospitals were trained in the diagnosis and management of VL patients. The program introduced the rapid diagnostic test (RDT rK39) and treatment with miltefosine for the management of VL at no cost for patients [5]. After dependence for six decades on one drug, sodium stibogluconate, Bangladesh has adopted a number of drugs including miltefosine, amphotericin B, liposomoal amphotericin B, and paromomycin in its weaponry against VL and post-kalaazar dermal leishmaniasis (PKDL). The diagnosis for VL and PKDL was scaled up to all the endemic sub-districts (upazilas). Adequate training of physicians and nurses at different levels along with free diagnostics and improved disease management led to almost zero mortality in the subsequent years (Fig. 1). Earlier reports estimated the VL burden in Bangladesh to be about 12,400-24,900 new cases annually with a mortality rate of $1.5 \%$ [1]. However, the number of cases has declined in the subsequent years and in 2011 it was 3,833 and in 2012 until October it was 1,463. The declining trend of VL burden as well as almost zero mortality rates can be explained by the successful activities of the national program complying with the strategic framework of the VL elimination program. The program has initiated an approach for early detection of cases of VL and PKDL and initiated indoor residual spraying in high VL endemic upazilas. Field activities of organizations such as the International Centre for Diarrhoeal Disease Research, Bangladesh, World Health Organization-TDR, and Medicins sans frontiers (MSF), Holland have also contributed to early detection of cases of VL and PKDL and complete treatment with highly effective drugs including AmBisome by MSF, Holland in the most highly VL-endemic sub-district (perhaps in the world), Fulbaria. The challenge for the program is how to maintain early case detection and complete treatment through continuous training programs for public health staff in sub-district hospitals, uninterrupted supply of diagnostics and drugs, and introduction of new single-dose treatment with liposomal amphotericin B (AmBisome) in collaboration with the World Health Organization as a first-line drug. Another challenge is that because the VL burden and case fatality rates have been declining, there is a possibility for VL to become less of a priority for the policy makers. This may result in funding constraints for human resources and logistics for case management. Thus, to keep the program activities beyond achieving the target of elimination, advocacy for the program by the national and international policy makers is necessary. The PKDL burden is highest in Bangladesh among the three countries of the elimination program. PKDL patients are a threat to the success of the elimination program because these patients are clinically healthy, do not seek medical care, harbor the parasite over the years, and can initiate new outbreaks of VL in the community. The duration of treatment of PKDL with sodium stibogluconate is long and involves 120 painful injections. Thus, the treatment compliance is frustratingly low. Unfortunately, a highly effective, safe, and shorter duration of treatment is not available for PKDL. A shorter 12-week treatment with miltefosine has been found to be effective and comparatively safe with better treatment compliance [6]. However, this report included only 26 PKDL patients and more studies are needed. Nevertheless, the national VL elimination program of Bangladesh has now introduced the 12-week oral miltefosine therapy for PKDL patients in July 2012. To date, about 100 adult PKDL patients have been treated with excellent tolerance and cure rates and data are currently being collected. Because miltefosine has some limitations, especially for its use in young children and women of child-bearing 


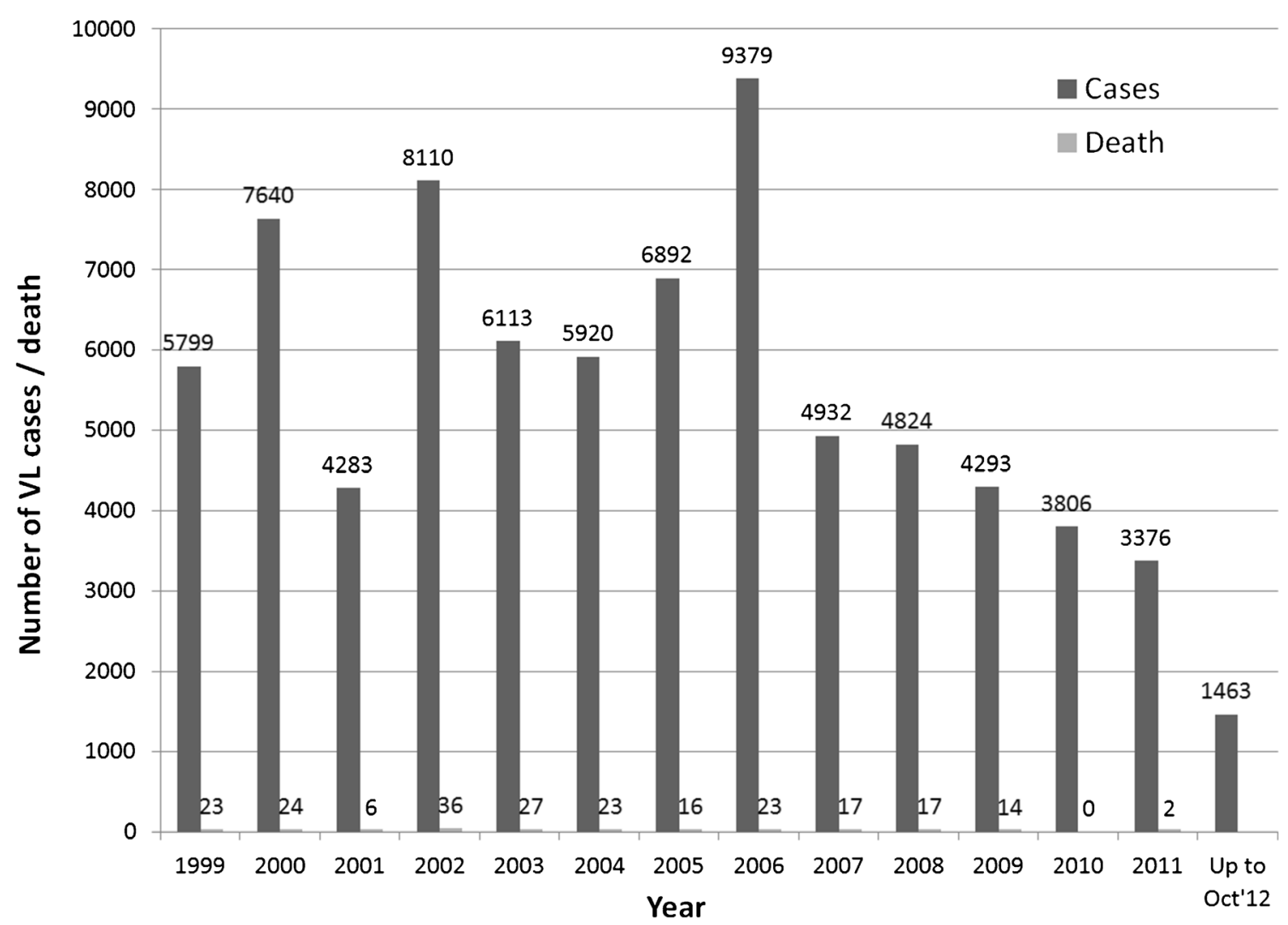

Fig. 1 Visceral leishmaniasis (VL) burden in Bangladesh since 1999

age, a better treatment regimen for PKDL is needed for the success of the elimination program. Safety and long-term efficacy of the 12-week treatment with miltefosine in children with PKDL needs to be investigated.

Integrated Vector Management

Although IVM was considered an important strategy for the VL elimination program in Bangladesh, there were no VL vector control activities in place until 2011 [7]. The national VL elimination program overcame major obstacles and initiated IVM in the VL-affected areas after successful piloting of indoor residual spraying with insecticides (IRS) in two unions (a union is an administrative geographic unit with a 25,000 population and 5,000 households on average) of Fulbaria, Mymensingh district. The program started blanket IRS in VL-affected villages in eight of the most highly endemic, VL sub-districts (Table 1). Although it is too early to know the impact of the IRS intervention, it appears that it is contributing to an improved reduction in VL burden (Fig. 1). Other factors including active case detection and treatment in the most highly endemic VL areas, and 'natural decline' of

Table 1 IRS activity of the visceral leishmaniasis elimination program of Bangladesh in 2011-2012

\begin{tabular}{lllll}
\hline District & Upazila & HH Target for IRS & Achievement of Pre-monsoon IRS & Achievement of Post-monsoon IRS \\
\hline Mymensingh & Muktagacha & 33,556 & 33,620 & 33,233 \\
& Trishal & 70,933 & 75,245 & 80,582 \\
& Fulbaria & 82,747 & 91,621 & 92,627 \\
& Goforgaon & 23,241 & 54,146 & 56,566 \\
& Bhaluka & 52,876 & 53,588 & 55,598 \\
Jamalpur & Madargonj & 52,396 & 23,235 & 26,450 \\
Tangail & Nagorpur & 9,607 & 9,510 & 9,607 \\
Khulna & Terokhada & 5,774 & 5,514 & 5,990 \\
Total & & & 346,746 & 360,659 \\
\hline
\end{tabular}

$H H$ household, IRS indoor residual spraying with insecticides 
Table 2 Long-lasting insecticide-treated bed-nets for integrated vector management in Bangladesh

\begin{tabular}{lllll}
\hline S/N & District & Upazila & Total KA and PKDL cases(2008) & No. of LLINs distributed \\
\hline 1 & Mymensingh & Fulbaria & 4,403 & 8,832 \\
2 & & Trishal & 1,843 & 3,800 \\
3 & Gafargaon & 951 & 1,812 \\
4 & Muktagacha & 875 & 1,254 \\
5 & & Bhaluka & 728 & 1,256 \\
6 & & Madarganj & 415 & 830 \\
7 & Jamalpur & Nagarpur & 143 & 300 \\
8 & Tangail & Terokhada & 136 & 272 \\
Total & Khulna & & 8,663 & 17,326 \\
\hline
\end{tabular}

$S / N$ serial number, $K A$ kala-azar, $L L I N$ long-lasting, insecticide-treated nets, $P K D L$ post-kala-azar dermal leishmaniasis

disease may also contribute to the reduction of VL burden. A trend analysis can be undertaken to investigate the impact of the IVM program and other activities against the disease. IVM usually comprises various interventions for vector control in combination, depending on suitability of their implementation. Currently, the efficacy of other components of IVM other than IRS is either disputable or not studied. Examples include: efficacy of long-lasting, insecticide-treated nets (LLINs) on vector control and disease transmission $[8,9]$ and the impact of behavioral change communication (BCC) on vector control. LLINs have been demonstrated to reduce sand fly density as shown in several studies $[10,11]$. As a result, a program of distributing LLINs to all households with VL and PKDL cases has been initiated to reduce transmission within household members. This is a unique intervention so far in the Indian sub-continent. A well-designed cohort study is now needed to evaluate the impact of the LLINs on VL transmission in these patient households. The program has now distributed almost 18,000 LLINs (Table 2) and thousands more are planned for distribution (Table 3).

Thus, the IVM of the Bangladesh VL elimination program comprises IRS and the distribution of LLINs. Despite significant achievement for the implementation of IVM, the program also has weaknesses and challenges. Weaknesses include: lack of regular vector surveillance, a weak reporting system, lack of external quality control of insecticide, dependence of IRS only on deltamethrin, lack of back-up regulatory authority-approved insecticides for VL vector control, weak participation of other stakeholders, and inadequate BCC intervention for VL vector control. Challenges of IVM include: insufficient funds, insufficient number of entomologists, knowledge gap about VL vector bionomics, and knowledge gaps about the efficacy of other insecticides and the most costeffective strategy for distribution of the LLINs to endemic areas. The program requires support from the national and international policy makers and donors to overcome these challenges.

There are also other opportunities for IVM in Bangladesh. $\mathrm{VL}$ is highly endemic in a few districts of Bangladesh and this will favor implementation of IVM in a cost-effective way. Except for deltamethrin, no other pyrethroids have been used for vector control in the country. The elimination program should have additional World Health Organization-approved insecticides in hand if sand flies eventually become resistant to deltamethrin. Another opportunity is that the bed-net impregnation program with slow-release insecticide tablets was

Table 3 Future plan for integrated vector management in Bangladesh for visceral leishmaniasis control

\begin{tabular}{|c|c|c|}
\hline $\begin{array}{l}\text { Hyper-endemic } \\
\text { Upazila }\end{array}$ & $\begin{array}{l}\text { Pre- and post-monsoon IRS/year } \\
\text { LLIN distribution: in first round, last } 5 \text { year, patients and routine } \\
\text { distribution to the current patients }\end{array}$ & $\begin{array}{l}\text { During April to May and September to October for } 3 \text { consecutive } \\
\text { years } \\
\text { First round: during September to October } 2012 \text { and routine } \\
\text { distribution to the patients up to } 2015\end{array}$ \\
\hline $\begin{array}{l}\text { Moderate- } \\
\text { endemic } \\
\text { Upazila }\end{array}$ & $\begin{array}{l}\text { Camp followed by focal spray (selected village) } \\
\text { IRS (blanket spray to all } \mathrm{HH} \text { of the selected villages) } \\
\text { LLIN distribution: same as hyper-endemic Upazilla }\end{array}$ & $\begin{array}{l}\text { May to June and November to December for } 3 \text { consecutive years } \\
\text { Same as hyper-endemic Upazilla }\end{array}$ \\
\hline $\begin{array}{l}\text { Low-endemic } \\
\text { Upazila }\end{array}$ & Camp followed by focal spray (selected Village) & Same as moderate-endemic Upazilla \\
\hline
\end{tabular}

$H H$ household, IRS indoor residual spraying with insecticides 


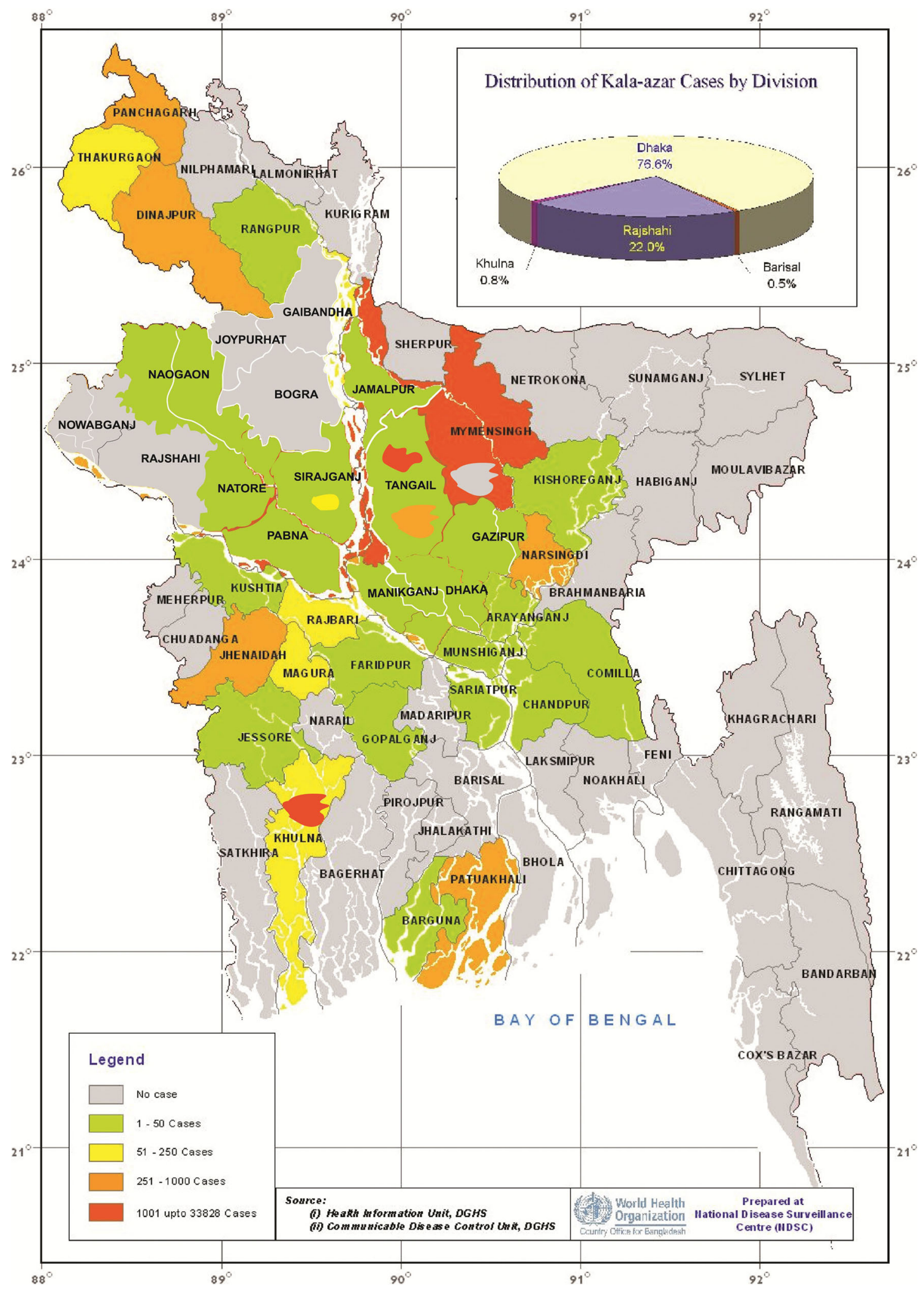

Fig. 2 Visceral leishmaniasis-affected districts in Bangladesh

shown to be effective for the reduction of sand fly density for a comparatively long period [11]. Thus, the program could compliment the IRS program with a mass bed-net impregnation program. 
Effective Disease Surveillance Through Passive and Active Case Detection and Vector Surveillance

Effective disease and vector surveillance is important for success in controlling any vector-borne disease of public health importance. The Disease Control Unit under the Directorate General of Health Services Bangladesh had established a surveillance system based on passive VL case reporting in the sub-district hospitals even before the initiation of the VL elimination program (Fig. 1). The major weakness of this surveillance program is under-reporting. Nevertheless, this is the only cost-effective surveillance system for the program to evaluate the disease situation in the country. To improve the disease surveillance system, in 2011, the program initiated a camp approach for active detection of VL and PKDL cases in endemic villages. The camp approach has been found to be the most cost-effective intervention for active detection of cases with VL and PKDL [12]. One of the remarkable successes of the program is that for the first time, all VL endemic villages are mapped and stratified according to their VL endemicity (Fig. 2).

Like disease surveillance, vector surveillance also needs improvement. Repeated entomological surveys have been performed during the piloting of IRS in the Fulbaria subdistrict and this can be taken as a sentinel site for vector surveillance. More areas now must be included for vector surveillance. The challenges for successful vector surveillance are constraints with human resources, insufficient number of entomologists, and lack of funds and logistics. There is a need for capacity building for vector surveillance and entomological research, particularly for sand fly insectariums, laboratories for testing insecticide susceptibility, and periodic internal quality control of insecticides.

\section{Social Mobilization and Building Partnerships}

Success of the national VL elimination program is not possible without the active participation of the community. Among the three countries, community knowledge and attitude was poorest in Bangladesh [13]. Current health education and social mobilization through billboard and leaflets are not sufficient to increase community awareness about VL. It is unknown what the most appropriate tools for health education are, especially for BCC. The program should pay more attention to the selection of $\mathrm{BCC}$ tools and to conducting periodic $\mathrm{BCC}$ intervention. $\mathrm{BCC}$ is a process that motivates people to adopt and sustain healthy behaviors and lifestyles. Sustaining healthy behavior usually requires a continuing investment in $\mathrm{BCC}$ as a part of an overall health program, which is a major challenge for the national program. Building partnerships with national NGOs, and national and international donor agencies is crucial. Currently, partnerships do exist for pilot interventions with MSF Holland, Institute For Epidemiology, Diseases
Control and Research (IEDCR), International Centre for Diarrhoeal Disease Research, Bangladesh, Shaheed Suhrawardy Medical College (SSMC), World Health Organization-The Special Programme for Research and Training in Tropical Diseases (TDR), Institute for One World Health (iOWH), Drugs for Neglected Diseases initiatives (DNDi), and Program for Appropriate Technology in Health (PATH). Despite these partnerships, the national VL elimination program is totally financed by the Ministry of Health and Family Welfare, Bangladesh and does not receive sufficient support from the international donor organizations. Strong advocacy for the sustainability of existing activities of the program as well as for the start of new activities such as $\mathrm{BCC}$ is required.

\section{Clinical and Operational Research}

This is one of the most successful areas of the national VL elimination program in Bangladesh and includes the clinical trials with miltefosine [14], combination drug therapy, and feasibility studies for single-dose AmBisome at the sub-district level. Trials have also been conducted with different vector control methods and studies for better diagnostic tools for VL and PKDL. Additional research activities on PKDL are planned. However, the program should consider additional research activities such as a follow-up epidemiological survey of VL and PKDL burden compared with the baseline survey performed in 2009. Research to identify cost-effective larvicidal methods, efficacy trial with new insecticides, and other vector control methods, and treatment trials for PKDL is also required.

Another major success of the program is the establishment of a Kala-azar research center at the Surja Kanta (SK) Hospital in collaboration with Japan International Cooperation Agency (JICA), Japan, Drugs for Neglected Diseases initiatives (DNDi), and the International Centre for Diarrhoeal Disease Research, Bangladesh. This hospital will treat VL relapse cases, VL cases with complications, and patients with PKDL. The research center at the SK Hospital is open to all researchers who are interested in conducting studies on VL and PKDL.

\section{Conclusion}

The national VL elimination program has experienced considerable success as a result of its operational research activities in early case detection and complete treatment, IVM, and clinical research. However, the program needs to strengthen its activities for effective surveillance, $\mathrm{BCC}$, monitoring, and evaluation. The main constraints for the program moving forward are insufficient human resources, funds, and logistics. These constraints may be overcome through building a 
partnership with national and international donor agencies and advocacy institutions, and by working closely with policy makers to ensure this successful program is sustainable.

Acknowledgments The authors acknowledge our gratitude to the District Civil Surgeons of the VL endemic districts of Bangladesh, Heads of the Upazila Health Complex, and Statistical Technician at Upazila Hospitals for their contribution in data collection and data compiling. We also wish to thank Greg Matlashewski for his valuable input in preparing this article.

\section{Compliance with Ethics Guidelines}

Conflict of Interest Be-Nazir Ahmed, Shah Golam Nabi, Mizanur Rahman, Shahjada Selim, Ariful Bashar, Md. Mahbubur Rashid, Fahima Yeasmin Lira, Tanveer Ahmed Chowdhury, and Dinesh Mondal declare that they have no conflict of interest.

Human and Animal Rights and Informed Consent This article does not contain any studies with human or animal subjects performed by any of the authors.

\section{References}

1. Alvar J, Vélez ID, Bern C, Herrero M, Desjeux P, Cano J, et al. Leishmaniasis worldwide and global estimates of its incidence. PLoS One. 2012;7(5):e35671.

2. World Health Organization: Regional Technical Advisory Group on Kala Azar Elimination. Report of the third meeting, Dhaka, Bangladesh. Dhaka: World Health Organization, SEARO 2009.

3. World Health Organization. Control of the leishmaniases. Report of a meeting of the WHO Expert Committee on the Control of Leishmaniases, Geneva, 22-26 March, 2010. WHO Technical Series 949.

4. World Health Organization. Regional Technical Advisory Group on Kala-azar Elimination. Report of the first meeting, Manesar, Haryana, 20-23 December 2004. New Delhi: Regional Office for South-East Asia; 2005.
5. Rahman R, Bangali M, Kabir H, Naher FB, Mahboob S. Kala-azar situation in Bangladesh. In: Hossain M, editor. Natinal Guideline and Training Module for Kala-azar Elimination in Bangladesh. 1st ed. Dhaka: CDC, DGHS, Ministry of Health and Family Welfare, Government of the Peoples Republic of Bangladesh; 2008.

6. Ramesh V, Katara GK, Verma S, Salotra P. Miltefosine as an effective choice in the treatment of post-kala-azar dermal leishmaniasis. Br J Dermatol. 2011. doi:10.1111/j.1365-2133.2011.10402.x.

7. Mondal D, Alam MS, Karim Z, Haque R, Boelaert M, Kroeger A. Present situation of vector-control management in Bangladesh: a wake up call. Health Policy. 2008;87(3):369-76. doi:10.1016/j. healthpol.2008.01.011.

8. Picado A, Dash AP, Bhattacharya S, Boelaert M. Vector control interventions for visceral leishmaniasis elimination initiative in South Asia, 2005-2010. Indian J Med Res. 2012;136(1):2231.

9. Picado A, Singh SP, Rijal S, Sundar S, Ostyn B, Chappuis F, et al. Longlasting insecticidal nets for prevention of Leishmania donovani infection in India and Nepal: paired cluster randomised trial. BMJ. 2010;341:c6760. doi:10.1136/bmj.c6760.

10. Picado A, Das ML, Kumar V, Kesari S, Dinesh DS, Roy L, et al. Effect of village-wide use of long-lasting insecticidal nets on visceral Leishmaniasis vectors in India and Nepal: a cluster randomized trial. PLoS Negl Trop Dis. 2010;4(1):e587. doi:10.1371/journal.pntd. 0000587.

11. Mondal D, Chowdhury R, Huda MM, Maheswary NP, Akther S, Petzold M, et al. Insecticide-treated bed nets in rural Bangladesh: their potential role in the visceral leishmaniasis elimination programme. Trop Med Int Health. 2010;15(11):1382-9. doi:10.1111/j. 1365-3156.2010.02635.x.

12. Singh SP, Hirve S, Huda MM, Banjara MR, Kumar N, Mondal D, et al. Options for active case detection of visceral leishmaniasis in endemic districts of India, Nepal and Bangladesh, comparing yield, feasibility and costs. PLoS Negl Trop Dis. 2011;5(2):e960. doi:10. 1371/journal.pntd.0000960.

13. Mondal D, Singh SP, Kumar N, Joshi A, Sundar S, Das P, et al. Visceral leishmaniasis elimination programme in India, Bangladesh, and Nepal: reshaping the case finding/case management strategy. PLoS Negl Trop Dis. 2009;3(1):e355. doi:10.1371/journal.pntd.0000355.

14. Rahman M, Ahmed BN, Faiz MA, Chowdhury MZ, Islam QT, Sayeedur R, et al. Phase IV trial of miltefosine in adults and children for treatment of visceral leishmaniasis (kala-azar) in Bangladesh. Am J Trop Med Hyg. 2011;85(1):66-9. doi:10.4269/ajtmh.2011.10-0661. 